\title{
Interacting viscous instabilities in microfluidic systems
}

\author{
Thomas Cubaud $* a$ and Thomas G. Mason $* b$ \\ Received 18th April 2012, Accepted 18th June 2012 \\ DOI: $10.1039 / \mathrm{c} 2 \mathrm{sm} 25902 \mathrm{~h}$
}

\begin{abstract}
We discuss the formation, evolution, and stability of microfluidic flows involving two or more miscible fluids that have different viscosities. When two liquids that have widely different viscosities are injected into a rigid microfluidic device, their flow streams can naturally rearrange to form lubricated threads or stratified flows depending on the geometry and history of injection. An overview of two-fluid and threefluid flow configurations in microchannels having square cross-sections is given for a variety of injection geometries. Miscible viscous fluid threads in confined microsystems can experience a range of viscous instabilities, such as folding and swirling. We show that microfluidics can be used to cause two or more instabilities to interact and co-evolve in diverging microchannels, thereby creating a variety of complex flow patterns.
\end{abstract}

\section{Introduction}

Beyond advancing the miniaturization of biotechnologies through lab-on-a-chip applications, microfluidic devices provide versatile and powerful platforms for studying basic aspects of fluid mechanics and rheology. Simple versions of microfluidic systems have been used for many decades in probing and exploring a variety of complex fluids and multiphase flows. Examples of small-scale flow geometries include thin-gap parallel-plate Hele-Shaw cells, ${ }^{1}$ interaction chambers in hard microfluidic homogenizers, ${ }^{2}$ thin-gap Couette shear cells, ${ }^{3-6}$ and capillary tubes having various shapes and sizes. ${ }^{7-9}$

${ }^{a}$ Department of Mechanical Engineering, Stony Brook University, New York, 11794, USA. E-mail: thomas.cubaud@stonybrook.edu

${ }^{b}$ Department of Chemistry and Biochemistry, Department of Physics and Astronomy, University of California, Los Angeles, California 90095, USA.E-mail:mason@chem.ucla.edu
More recently, microfluidic devices have increasingly harnessed the power of lithography, ${ }^{10}$ yielding control over multiple fluid injections and complex flow geometries. These developments have enabled the fabrication of integrated biochemical sensors ${ }^{11,12}$ and a range of actuators, such as valves, heating elements, and electrical conductors for manipulating electrokinetic effects. ${ }^{13,14}$ Custom designed microfluidic cell geometries that provide optical access, when used in combination with highspeed microscopic imaging technology and digital control of fluid flows, yield new control over driving stresses and insight into highly complex flows in the absence of significant gravitational effects and from low to moderate Reynolds numbers, Re.

Microfluidics can be used to create a variety of fluid instabilities in a controlled manner. Interactions of flowing complex fluids within solid microgeometries yield intriguing effects such as structural rearrangements of aqueous foams, ${ }^{15-17}$ breakup and coalescence of droplets, ${ }^{18-23}$ and the formation of multiple emulsions and compound drops. ${ }^{24-26}$ In addition to producing

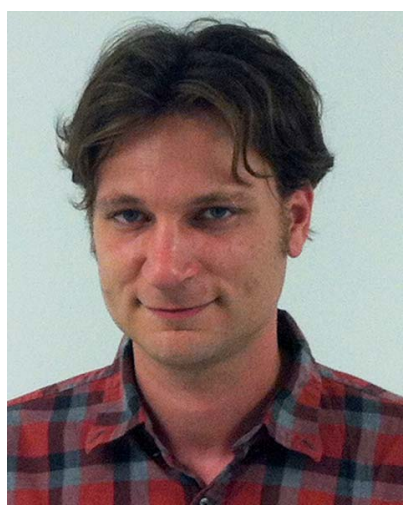

Thomas Cubaud
Thomas Cubaud is an Assistant Professor of Mechanical Engineering at Stony Brook University. He received his Ph.D. from Paris-Sud University for his research at ESPCI, France, and he worked at UCLA as a postdoctoral research scientist. $D r$ Cubaud is the recipient of an NSF CAREER Award and three Gallery of Fluid Motion Awards from the American Physical Society, Division of Fluid Dynamics.

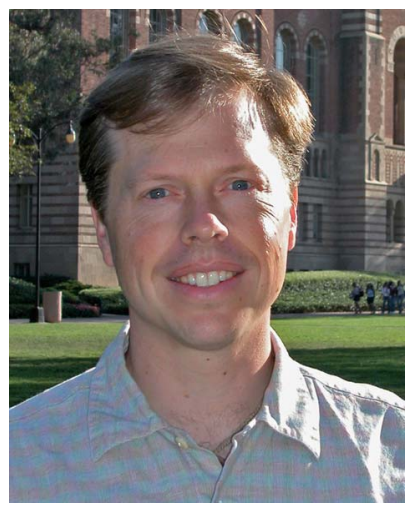

Thomas G. Mason
Thomas G. Mason is a Professor of Physical Chemistry and a Professor of Physics at UCLA. The inventor of probe-based thermal "passive" microrheology, Mason pursues research in structure-dynamicsflow relationships and novel forms of soft matter. He is a fellow of the American Physical Society, has received an NSF CAREER Award, and shares two Gallery of Fluid Motion Awards with Prof. Cubaud. 
some of the classic instabilities that occur between two fluids in bounded systems, such as viscous fingering (i.e., Saffman-Taylor) ${ }^{27-31}$ and capillary (i.e., Rayleigh-Plateau) ${ }^{32-34}$ instabilities, microdevices have provided insight into elastic instabilities ${ }^{35-40}$ for non-Newtonian fluids at low Re, and inertial instabilities ${ }^{\mathbf{4 1 - 4 3}}$ for moderate Re. Among the experimental challenges that exist in the area of soft matter microfluidics, perhaps the most important ones are: developing advanced and efficient lithographic techniques that facilitate the construction of 3D microchannels, fabricating robust microdevices that can withstand large injection pressures for handling highly viscous fluids, and devising methods for rapidly mixing viscous materials at the small scale.

The flow of miscible fluid streams having similar viscosities has been studied in microfluidics for the formation of transverse and longitudinal diffusion gradients. ${ }^{44-46}$ A useful technique for manipulating fluids consists in hydrodynamically focusing a sample stream using sheath flows. ${ }^{47}$ The initial size and spread of parallel streams have been experimentally and theoretically examined for a variety of channel designs and flow rates. ${ }^{48-52}$ Mixing two different streams at the small-scale is rather challenging since it typically occurs through the relatively slow process of diffusion. This situation is in contrast with large-scale systems that rely on turbulence to blend fluids. To circumvent this limitation, two main types of microsystems have been developed: (a) active micromixers, which involve additional energy sources or moving parts, and (b) passive micromixers, which rely on flow properties and microgeometries. ${ }^{53}$ In general, the objectives of micromixers are to increase the interfacial area between fluids to reduce the diffusional path and to exploit the concept of chaotic advection ${ }^{54}$ while minimizing both the fluid residence time and the device dimensions. Mixing thicker soft materials with thinner solvents (i.e. fluids having a large viscosity contrast) in microchannels is very complex because the local viscosity evolves as fluids mix and propagate through the microchannel network, and only a few methods - including acoustically induced bubbles, ${ }^{55}$ splitting-and-recombination processes $^{56}$ and viscous folding instabilities ${ }^{57}$ - have been investigated to date.

In this article, we provide a perspective on the use of microfluidic technology for generating and studying interacting viscous instabilities, focusing on miscible and Newtonian fluids having large viscosity contrasts and flowing at low $\mathrm{Re} \leq O(1)$. We show how the concept of self-lubrication ${ }^{5-60}$ between differing-viscosity fluids can be utilized in microchannels to form single or multiple threads of more viscous liquids that are lubricated by less viscous liquids which co-flow and envelop the threads. This typical core-annular flow configuration facilitates both transport and manipulation of high-viscosity fluids at the small scale. Using the superposition principle of low Reynolds numbers flows, we show that threads can be used to build a variety of 3D fluid arrangements within quasi-2D microchannels. Depending on material properties, injection geometries, and rates, these threads and stratified flows then can undergo different types of instabilities that can be used to increase the fluid-fluid interfacial area, thereby reducing the diffusional path and facilitating mixing.

Viscous fluids in slender geometries that impinge on surfaces are known to buckle through viscous folding and coiling instabilities. ${ }^{61-63}$ Such viscous instabilities result from both the large viscosity coefficients of fluids and their structural configurations, and can be produced at low $\mathrm{Re}$ in microchannels between miscible Newtonian fluids (i.e., in the absence of interfacial tension). Therefore, these viscous instabilities are different than the elastic, inertial, and capillary instabilities previously mentioned. We illustrate the complex pattern formation accompanying viscous instabilities through a series of experimental results involving miscible Newtonian silicone oils injected into hard silicon-glass microfluidic devices.

When two fluids are considered, we discuss the folding instability caused by the deceleration of a viscous thread surrounded by thin miscible fluid in a diverging slit microchannel. The folding instability can undergo a period doubling route to chaotic folding. ${ }^{57}$ Ultimately, through a combination of convection and diffusion, the liquids intermix and the observed folding pattern, due to the optical refractive index difference between the liquids, disappears further downstream. In a different scenario, exploiting the laminar properties of microflows, a viscous thread is formed in a manner that enables control over the distance between the thread and the sidewall of a microchannel that has a square cross-section. As the thread begins to approach the sidewall, the gradient in velocity within the outer less viscous oil exerts a torque on the thread, causing it to undergo a "swirling" instability. ${ }^{64}$

When three miscible fluids are injected and flow co-currently from a channel having a square cross-section to a diverging channel, the number and complexity of flow patterns formed become quite high. We show that several types of hydrodynamic instabilities can be forced to occur, interact, and compete simultaneously. For instance, three different miscible liquids can be fed into a set of two cross-channels in series, and by controlling the various injection points, viscosities, and flow rates, different complex patterns, involving combinations of selflubrication, folding, swirling, and fingering instabilities, emerge. We demonstrate that it is possible to form viscous fingers using steady fluid injections of fully miscible fluids; yet, these fingers are superimposed on a more complex flow pattern. Thus, some patterns we report are reminiscent of the classic Saffman-Taylor instability that occurs when a less viscous fluid displaces a more viscous fluid at rest. However, in the more complex microfluidic geometries that we have explored, coupling between timedependent and space-dependent complex fluid flows and viscous material properties can lead to two or more interacting instabilities. The resulting dynamical patterns that form are both complex and interesting, and can provide a wealth of information about the interplay and evolution of viscous fluid instabilities in microfluidic systems.

\section{Experimental methods}

To produce and examine small-scale multi-fluid flows involving two or more highly viscous liquids, it is desirable to avoid problems that can occur when injecting these liquids into soft microfluidic devices (e.g. unwanted deformation and separation of sandwich layers caused by high injection pressures). By contrast, hard microfluidic modules, made of silicon and glass, provide robust resistance to high injection pressures, while ensuring good optical access, and are therefore preferred. 
Silicon-based nanofabrication techniques offer the possibility to construct non-deformable microchannels, which are used to perform precise measurements and obtain quantitative data. Double-side polished silicon layers are deep-reactive-ion-etched through their entire thickness $(h=100 \mu \mathrm{m})$ to form the microchannel sidewalls. The top and bottom walls are two glass plates, and these are anodically bonded to the etched silicon piece to fully seal the duct. A collimated light source is placed on one side of the device and flows are monitored with a high-speed camera through a high-magnification lens having a very long working distance that is located on the other side of the channel. This optical method visualizes flows using transmitted light in bright field and overall enables the imaging of curved miscible fluid interfaces (where there is a significant difference in refractive index) in the absence of added dye. The test fluids consist of pure silicone oils (poly-dimethylsiloxane or PDMS) having different molecular weights. Such simple liquids are practical because a change in viscosity does not significantly affect other properties, such as surface tension. According to the Stokes-Einstein relation, ${ }^{65}$ the molecular diffusivity, however, is affected by the molecular weight and low-viscosity oils are strongly diffusive with respect to their high-molecular weight counterparts. ${ }^{66}$ Fluids are injected into the device and steady flow rates are controlled using several independent syringe pumps.

The microgeometries of the hard microfluidic devices are designed to create structured and stable viscous flows near the injection region; these flows can then be destabilized in interesting ways further downstream from the injection region. In particular, we focus on basic micro-duct geometries having symmetric injection ports, including: (1) parallel flows with straight channels, which are either compact (square in crosssection) or planar (high aspect ratio Hele-Shaw cell with sidewalls), and (2) extensional flows (such as diverging and converging sections). Fluids are injected using various symmetric hydrodynamic focusing sections connected to a square channel discharging to a diverging channel characterized by a divergence angle $\alpha$ [Fig. 1]. Low Re flows (i.e., creeping or Stoke flows) typically have a very small entrance length, ${ }^{67}$ and the superposition principle for different velocity fields is evident. ${ }^{68} \mathrm{Here}$, we are primarily interested in flow regimes that occur for steady fluid injections (i.e., constant flow rates) into constrained microgeometries. In particular, we describe miscible multi-fluid flows having either a "sequential" fluid injection scheme (i.e., injection via cross-channels in series) with three injection ports [Fig. 1(a)] or a simultaneous fluid injection scheme with four injection ports to minimize the device footprint [Fig. 1(b)]. Ports and liquids are indexed symmetrically from the center of the channel outward. Compared to a single microfluidic focusing section, "port 1" corresponds to the central channel where the liquid $L 1$ of dynamic viscosity $\eta_{1}$ is injected at a volumetric flow rate $Q_{1}$, "port 2" corresponds to the side-channels where liquid $L 2$ of viscosity $\eta_{2}$ is injected with the total flow rate $Q_{2}$. The same index-labeling rule applies for additional liquids and ports.

These devices permit the permutation of injection of lower and higher viscosity liquids at controlled rates into the various injection ports, in a systematic and symmetric fashion, enabling the exploration of morphology and stability of multi-fluid flows as a function of initial conditions (i.e., injection order). A particularly attractive feature of highly viscous multiphase flows

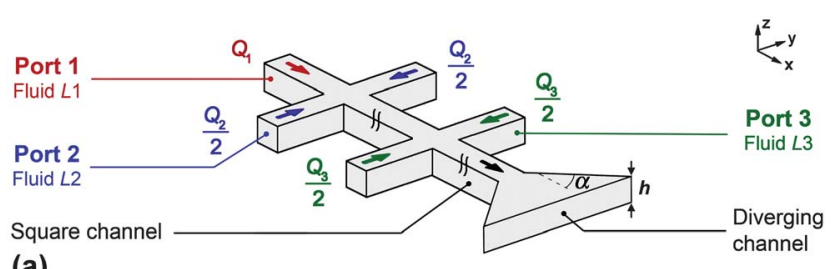

(a)

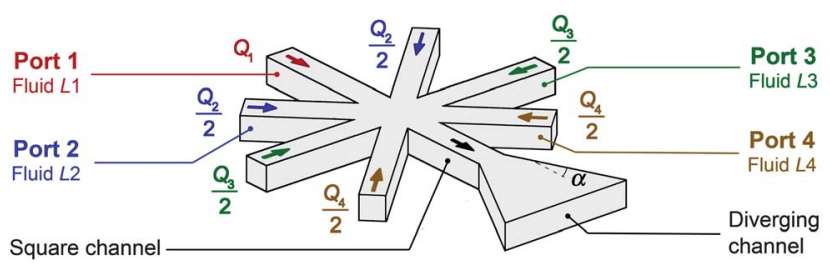

(b)

Fig. 1 Schematics of symmetrical injection devices for the formation of multi-fluid flows in square-cross-section microchannel connected to a diverging channel of angle $\alpha$. A slit channel and an outlet port further downstream, well beyond the diverging channel region, is not shown. (a) Dual hydrodynamic focusing sections in series. The length of the square channels between section is not to scale (three injection ports, $\alpha=45^{\circ}$ ). (b) Simultaneous injection scheme in a "spider-type" connection (four injection ports, $\alpha=15^{\circ}$ ).

is the possibility of initiating self-lubrication flow phenomena to build elaborate $3 \mathrm{D}$ fluid configurations using simple 2D designs, which are more suitable for standard lithographic methods that only require a minimal technical overhead (i.e., because fluid injections normal to the plane of the device are not necessary). Optical imaging is performed using a fast digital camera, and 2D movies of the evolving pattern formation resulting from isolated or interacting instabilities are recorded before the miscible liquids completely intermix. Although the complex 3D viscosity and velocity fields associated with the unstable flows within the microgeometries cannot be deconvolved from simple 2D movies, the contrast in refractive index of the different liquids prior to full intermixing provides a high level of insight into the flow fields arising from the instabilities.

\section{Two-fluid flow instabilities}

\subsection{Propagating fluid arrangements in a square channel}

Depending upon the details of the injection geometry, the fluids chosen for each port, and the injection rates, it is possible for lower-viscosity liquids to fully encapsulate higher-viscosity liquids. Indeed, microflows made of parallel layers of fluids having significantly different viscosities tend to self-organize into flow structures that minimize dissipation. Lower-viscosity fluid components tend to migrate into regions having higher shear stresses, i.e., near the solid walls; whereas, thicker, higher-viscosity fluid components tend to migrate into regions having lower shear stresses, i.e., near the channel axis. This yields the formation of self-lubricated threads of higher-viscosity fluids surrounded by coflowing lower viscosity fluids. This phenomenon, however, depends on the initial conditions (i.e., injection scheme, geometry, and rates and also upstream flow history). In practice, although microchannels have finite lengths, useful flow arrangements, such as threads or other stratified flows, can still be produced over short 
propagation distances from the injection region. In the following, we examine the flow morphology in the "square channel" region, which lies downstream from the injection region, yet upstream from the diverging channel region [Fig. 1].

We turn our attention to the formation of two-fluid flows in a square channel of height $h$ using a variety of symmetric injection ports. The most elementary configuration corresponds to a single focusing section with only two liquids, one being higherviscosity, or "viscous" $(V)$, and the other being lower-viscosity, or "fluid" (F) [Fig. 2(a) and (b)]. When the thicker fluid $V$ is injected in the central channel and the less viscous fluid $F$ is introduced in the side-channels, we observe the formation of a single viscous thread of diameter $\varepsilon$ lubricated by the less viscous fluid [Fig. 2(a)]. Using both miscible and immiscible Newtonian fluid pairs, ${ }^{34,69}$ this regime occurs for relatively large viscosity contrasts $\chi=\eta_{1} / \eta_{2}=\eta_{V} / \eta_{F}>15$. Optical measurements of the central stream width $\varepsilon$ show good agreement with the asymptotic solution $\varepsilon / h=(\varphi / 2)^{1 / 2}$, where the flow rate ratio is $\varphi=Q_{1} / Q_{2}$. This scaling form can be analytically deduced by considering a circular duct when $\chi \gg 1$ and $\varepsilon / h \ll 1$. The absence of $\chi$ in this relationship gives evidence of the full lubrication of the viscous thread. This regime corresponds to an asymptotic behavior where the liquid $V$ forms a core that undergoes plug flow (i.e. has a flat velocity profile); this core is convected in the parabolic Poiseuille flow of $F$.

The complementary situation, characterized by the fluid $F$ being introduced in the central channel and the fluid $V$ injected in the side-channels, yields a completely different scenario, since $V$ remains in its initial location near the walls. In this case, the flowing liquids simply form parallel layers and the fluid $F$ forms a central stream of width $d$ with the high-viscosity fluid in creeping flow near the sidewalls. Recent experimental findings ${ }^{70}$ showed that in square microchannels such viscous-stratified flows display a central stream width such as $d / h=\left[1+\left(\varphi \chi^{1 / 2}\right)^{-1}\right]^{-1}$. This

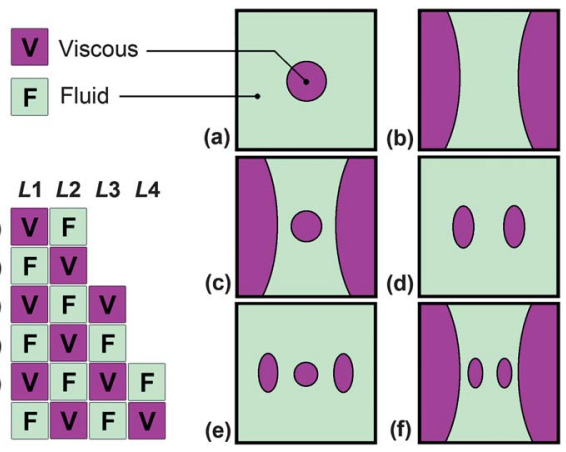

Fig. 2 Basic configurations of two co-flowing fluids in square microchannels, beyond the injection region. Schematics are cross-sectional views of 3D fluid compositions ( $V$ : higher viscosity, $F$ : lower viscosity), propagating normal to the page in square microchannels, hypothesized from $2 \mathrm{D}$ experimental images taken from the top (i.e. perpendicular to the flow direction). In (a)-(f), top and side boundaries of squares are glass and silicon, respectively. Two ports: (a) lubricated thread, (b) viscositystratified flow. Three ports: (c) lubricated thread in the central stream of a viscosity-stratified flow (predicted, not inferred from observations), (d) two lubricated threads. Four ports: (e) three lubricated threads, (f) two lubricated threads in the central stream of a viscosity-stratified flow (predicted, not inferred from observations). empirical relationship is deduced from the solution of the Stokes equation in a Hele-Shaw geometry with a flat interfacial region: ${ }^{66}$ $d / h=\left[1+(\varphi \chi)^{-1}\right]^{-1}$. In square channels, however, the stress condition at the fluid interface must be modified similar to the $2 \mathrm{D}$ free-shear layer problem of parallel streams of different viscosities, ${ }^{71}$ and experiments have shown that $d$ scales with $\varphi \chi^{1 / 2}$ in square channels while $d$ scales with $\varphi \chi$ in plane channels. Selflubrication effects also yield an interfacial curvature as the less viscous fluid tend to occupy the high-shear region near the top and bottom walls [Fig. 2(b)]. The dependence of stream widths on fluid viscosities is useful for the development of miniature viscometers. $^{72}$

Using three and four injection ports with only two different miscible fluids provides a means of building elaborate fluid structures. The two basic flow configurations previously discussed can serve as a guide to test other possibilities and manipulate fluid arrangements at the microscale. For instance, the configuration " $V F V$ " is expected to give a single thread with viscous strata at the walls and configuration " $F V F$ " yields two threads [Fig. 2(c) and (d)]. Four injection ports form more complex patterns with either three threads [Fig. 2(e)] or two threads flowing in the central stream of a stratified flow [Fig. 2(f)]. The configurations associated with two and three threads have been tested experimentally using both sequential and simultaneous injection scheme. Using a mass conservation argument, ${ }^{64}$ it has been deduced that a thread propagating offcenter does not have a circular cross-section; instead, the thread has an ellipsoidal cross-section due to the velocity field in a square channel. In general, the observed thread widths are in good accord with the thread-forming principle when applied to a symmetric injection scheme at the small scale.

\subsection{Instabilities of viscous threads}

The lubricated thread configuration offers the advantages of controlling both the residence time and the interfacial area of streams of higher viscosity materials surrounded by co-flowing streams of low-viscosity "solvents" simply by adjusting the injection flow rates. A thread that is well aligned with the axis of a compact and straight microchannel is relatively robust and does not experience significant destabilizing stresses. However, in other cases, viscous instabilities occur. We have identified two basic instabilities for threads, namely (1) a folding instability ${ }^{57}$ that results when threads are subject to a compressive stress and deceleration along the flow direction in a diverging channel, and (2) a viscous swirling instability that results when a thread is significantly sheared in a velocity gradient of the less viscous fluid, i.e., near the channel's side-walls. ${ }^{64}$

3.2.1. Diverging flows: thread folding. Diverging slit microchannels constitute basic microfluidic designs and are important for scaling up processes (e.g., for recovering soft materials from microchannels). In extensional flows, the convective acceleration in the Navier-Stokes equation is non negligible, and dynamic stresses are exerted on fluid structures. For instance, a droplet typically elongates along the flow direction in a converging channel and deforms normal to the main flow direction in a diverging channel. ${ }^{73}$ Similarly, continuous slender structures deform in extensional flow fields and lubricated threads are 
observed to buckle in diverging channels [Fig. 3]. For moderate viscosity contrasts, a thread simply enlarges (i.e. expands its diameter) along the diverging flow direction, whereas for very large $\chi$, the thread's thickness remains essentially constant along the flow direction, and the thread buckles rather than dilates. ${ }^{74}$ The folding frequency is linearly related to the injection flow rate in the folding regime for large threads. ${ }^{57}$ Diverging channels also form an effective transition zone between compact channels, which facilitate thread lubrication, and planar channels, which favors viscosity-stratified flows. As a result, threads can experience a non-linear lubrication failure in the diverging channel. ${ }^{75}$

The folding instability also occurs for multiple threads. To examine the crossover between single and multiple thread folding, we progressively increase $Q_{1}$ to observe the transition from a single thread to two threads [Fig. 3(a)-(c)]. In the two-thread case, the fact that filaments are lubricated allows for manipulating their distance with the virtual interface between $Q_{1}$ and $Q_{3}$, which follows the relationship $d / h=\left[1+\left(Q_{1} / Q_{3}\right)^{-1}\right]^{-1}$ since the viscosity ratio between $L 1$ and $L 3$ is unity. For small $d$, the two threads are observed to phase-lock, i.e., they fold in tandem. Fig. 3(d) shows the formation and folding of threads using the channel design shown in Fig. 1(b). Overall, the folding instability increases the threads' surface areas, thereby facilitating faster intermixing of higher and lower viscosity liquids downstream.

3.2.2. Off-center threads in velocity gradients: swirling instability. Another basic viscous thread instability occurs in straight channels for lubricated filaments located near the solid walls. While exploring the range of parameters for two threads in a square channel, we observed the development of a shear-induced instability $^{64}$ for large thread separations $d$ and small thread

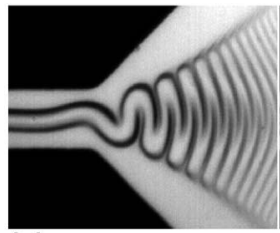

(a)

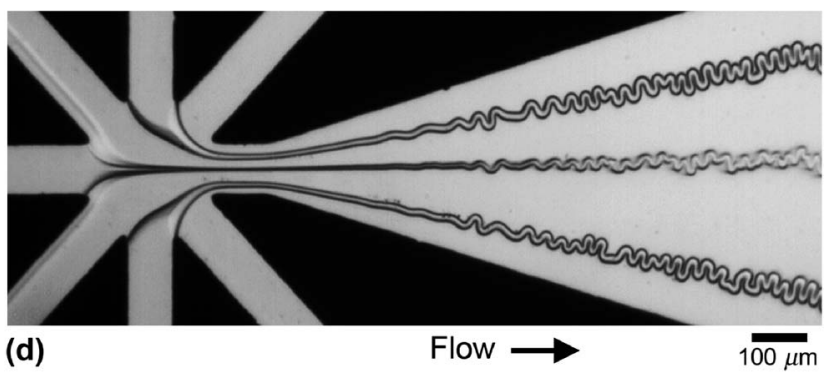

Fig. 3 Methods for creating single and multiple threads of a higher viscosity liquid, surrounded by a lower-viscosity liquid, that fold as they co-flow into diverging channels. The viscosity contrast between the lowand the high-viscosity fluids is $\chi=83$, flow rates are in $\mu 1 \mathrm{~min}^{-1}$. (a) Single folding for two ports, $Q_{1}=5, Q_{2}=13$ (adapted from ref. 57). (b) Two threads in close proximity fold in tandem (i.e. strongly phase-locked), $Q_{1}=5, Q_{2}=1, Q_{3}=10$. (c) Two well-separated threads folding, $Q_{1}=5$, $Q_{2}=5, Q_{3}=10$ (adapted from ref. 64). (c) Formation of three wellseparated threads by a spider-type injection that fold in the diverging region, $Q_{1}=1, Q_{2}=20, Q_{3}=2, Q_{4}=20$ (adapted from ref. 69). thickness $\varepsilon$. To represent the flow destabilization process, the velocity field of the less viscous fluid $F$ is displayed in Fig. 4(a). In the high-shear region (i.e., near the side-walls), the velocity differential on either side of the thread destabilizes its slender viscous structure over time and distance [Fig. 4(b)], yielding an evolving pattern that is reminiscent of the Kelvin-Helmholtz instability of a single planar interface. Although the deformation process is continuous, four basic steps are identified with (a) the development of the most dominant wavelength $\lambda$ along the thread, (b) the local accumulation of viscous fluid in "bulbs", (c) the thinning of the tails connecting bulbs, and (d) the detachment of bulbs from their neighbors to form independent viscous swirls. As expected for finite objects in a shear flow, swirls rotate during advection and can also pair up. Here, small Reynolds numbers have been investigated, and there is no significant drift in the low shear region.

Using a single fluid pair having large viscosity contrast $\eta_{V} / \eta_{F}=$ 83 , we have shown that the mean wavelength of destabilization $\lambda$ normalized by the thread diameter $\varepsilon$ is inversely proportional to the thread separation $d$ according to $\lambda / \varepsilon \approx 2.3 / d$. We have devised a simple scaling model for predicting this behavior based on the viscous-elastic analogy. ${ }^{76}$ Experiments involving a single thread aligned off-centered from the channel axis show a similar shear-induced swirling instability. ${ }^{69}$ This regime is important, since it enables the formation of discrete fluid elements (i.e., ephemeral swirls reminiscent of droplets) using miscible fluids in a continuous flow configuration.

\section{Three-fluid flow instabilities}

\subsection{Propagating fluid arrangements in a square channel}

In this section, we examine the multi-fluid flow configurations for a system composed of three miscible Newtonian fluids, one being (a) Viscous thread

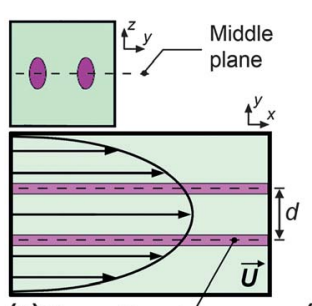

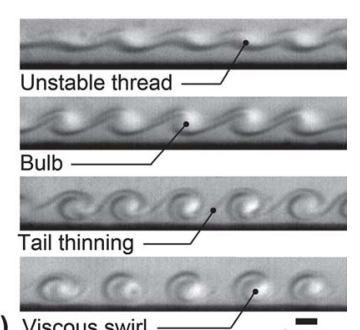

(b)

$20 \mu \mathrm{m}$
Fig. 4 Swirling instability of two miscible threads in a straight, squarecross-section microchannel, downstream from the injection region. (a) Schematic of the main velocity profile $U$ in the channel middle plane (lower part). Two lubricated threads (purple) near the channel axis remain relatively stable to shear-induced perturbations, but experience flow-induced torques due to the velocity gradient in the lower-viscosity liquid (light green). Hypothesized cross-sectional view of the square microchannel having two off-center threads, just after formation, prior to the development of the swirling instability further downstream (upper part). (b) Optical microscopic observations of the development of the viscous swirling instability for a miscible thread near the channel sidewalls, three injection ports, viscosity contrast $\chi=\eta_{V} / \eta_{F}=83$, flow rates in $\mu 1 \min ^{-1}: Q_{1}=20, Q_{2}=3, Q_{3}=20$. Images shown are closer to the injection region (top) and further downstream (bottom) where the growth of the instability causes the formation of uniform swirls (adapted from ref. 64). 
very viscous $(V)$, one being moderately viscous $(M)$, and one being less viscous, i.e. fluid $(F)$. Given the large number of potential injection scenarios, we restrict our discussion to a sequential fluid injection involving three ports [Fig. 1(a)]. As fluids and injection ports are distinguishable, their permutation leads to the six basic configurations displayed in Fig. 5. These configurations, labeled from (a) to (f), are hypothesized from the encapsulation mechanisms observed in two-fluid systems using the superposition principle of creeping flows.

In the two first configurations, (a) and (b), $F$ is injected near the sidewalls, which leads to the formation of compound threads having either a highly-viscous core wrapped by $M$, which in turn is enveloped by $F$ [Fig. 5(a)] or a thread of $M$ with threads of $V$ on both sides [Fig. 5(b)]. Although, theses flow arrangements are logically deduced from their two-fluid counterparts, their experimental behavior in a diverging channel corroborate our hypothesis since the configuration (a) leads to the symmetric folding of $V$ within a layer of $M$ surrounded by $F$, and configuration (b) produces the folding of the two side threads. ${ }^{69}$ Configurations (c) and (d) consist of injecting $M$ near the sidewalls. Configuration (c) has not been experimentally examined, but we speculate that such flow arrangement is a priori possible. The configuration (d), where two threads are located at the interface between $F$ and $M$ fluids, has been extensively investigated in laboratory experiments and leads to very intriguing dynamics that we describe as a case study in the next section. Finally, configurations (e) and (f) occur when $V$ is injected near the sidewalls. Assuming that the highly viscous liquids form effective "viscous walls", the proposed configurations are plausible for strongly stratified flows.

\subsection{Parallel flows}

In a straight square microchannel involving three different miscible fluids, the configuration shown in Fig. 5(d) displays a wide range of different dynamical behaviors. A series of
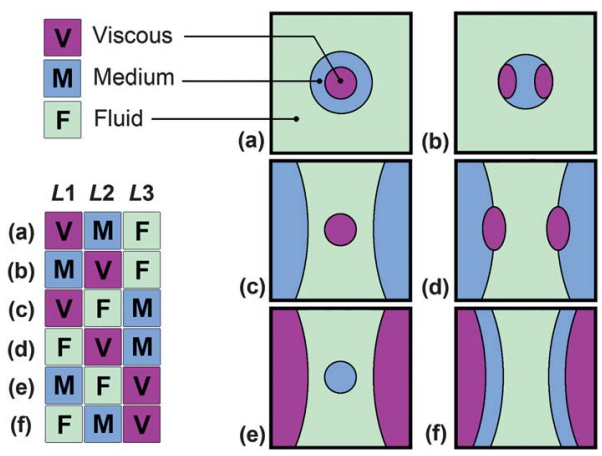

Fig. 5 Basic steady-state flow configurations of three fluids ( $V$ : highest viscosity, $M$ : medium viscosity, $F$ : lowest viscosity) in a square-crosssection microchannel, downstream from the injection region, propagating normal to the page, hypothesized from encapsulation mechanisms based on 2D observations of simpler two-fluid flows. (a) and (b) show different types of lubricated compound threads, (c) and (e) each show a lubricated thread in the central stream of a viscosity-stratified flow (predicted, not inferred from observations), (d) two threads at the interface of a viscosity-stratified flow, (f) double viscosity-stratified flow (predicted, not inferred from observations). experiments $^{77}$ using the microdevice shown in Fig. 1(a), has revealed that two viscous threads, made of $V$ ( $L 2$ with $\eta_{2}=$ $500 \mathrm{cP}$ ), are localized at the interface between the central stream, made of a thin diffusive fluid $F$ ( $L 1$ with $\eta_{1}=0.5 \mathrm{cP}$ ), and stratifications made of a medium viscosity fluid $M$ ( $L 3$ with $\eta_{3}=$ $6 \mathrm{cP}$ ). In this case, the main velocity field corresponds to a typical stratified flow with a faster-moving central region and a slowermoving creeping flow at the walls [Fig. 6(a)]. By virtue of our injection system, viscous threads are placed at the strongly sheared interface between $F$ and $M$ and can readily be destabilized by the swirling instability. In the stratified flow configuration, the less viscous fluid in the center effectively "slips" on the more viscous fluids, which changes the boundary conditions compared to a solid wall.

Fig. 6(b) shows the stability diagram for the threads of $V$ where the normalized average thread spacing $d / h$ is plotted as a function of the volume fraction of the viscous fluid $V, \alpha_{2}=Q_{2} /$ $\left(Q_{1}+Q_{2}+Q_{3}\right)$. The thread-thread distance $d$, which is measured as the average length between estimated thread centers, depends on the flow rate ratio, $Q_{1} / Q_{3}$, and $\alpha_{2}$ provides a measure of the threads' size. A typical experiment consists in fixing $Q_{2}$ and $Q_{3}$ and increasing $Q_{1}$. In this case, a single operating parameter can dramatically change flow morphologies. For the range of parameters investigated, we find that these highly structured flows present three basic configurations.

The stable configuration corresponds to a parallel viscositystratified flow and occurs for $d / h<0.33$. Threads propagate stably for small $d$ because the threads are located near the channel axis in the low-shear region. As $d$ increases, arrays of swirls are observed along the threads in the square channel [Fig. 6(c)-(ii)]. For dimensionless thread-thread spacings above a critical value of $d / h \sim 0.6$, experimental observations show very distorted flows that are characterized by a large central core that undulates and a variety of transient bursts in the channel. In this

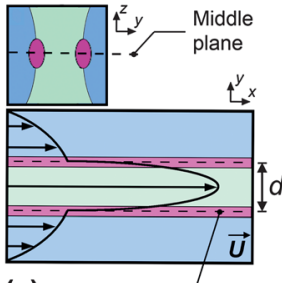

(a) Viscous thread

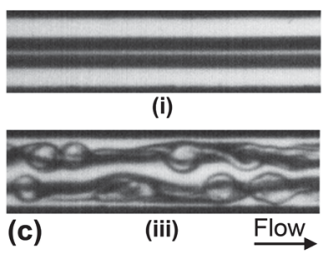

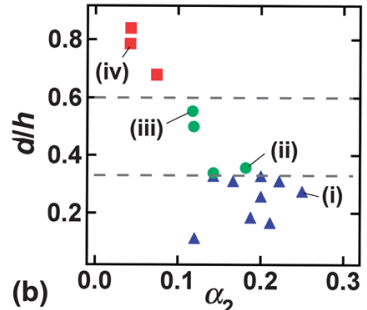

(b)

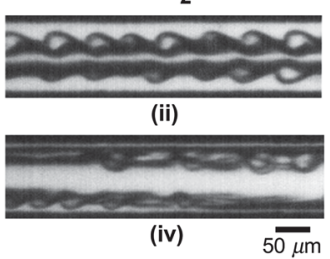

Fig. 6 Behavior of two threads of $V$ at the interface of a viscositystratified flow made of $F$ (central region) and $M$ (near the walls). (a) Schematic of the velocity profile $U$ in the middle plane. (b) Stability phase-diagram where the thread mean distance $d$ is measured as a function of the viscous liquid $V$ volume fraction $\alpha_{2}=Q_{2} /\left(Q_{1}+Q_{2}+Q_{3}\right)$ : stable flow $(\boldsymbol{\Delta})$, appearance of viscous swirls $(\bullet)$, and strongly unstable flows (ם). (c) Experimental micrographs taken at a distance $x / h \approx 20$ downstream from port 3, flow rates in $\mu 1 \mathrm{~min}^{-1}, Q_{2}=10, Q_{3}=25$ : (i) $Q_{1}=$ 15, (ii) $Q_{1}=20$, (iii) $Q_{1}=50$, and (iv) $Q_{1}=100$. 
case the unsteady nature of this flow results from the strong interplay between the large interfacial shearing between $F$ and $M$, and the strong development of the swirling instability near the walls. The transition between the viscous swirl flows and highly unstable flows is more apparent when examining the resulting flow in a diverging channel. Overall, the flow becomes unstable for large thread spacing $d$ and small thread volume fraction $\alpha_{2}$, which is essentially similar to off-center threads that experience torques driven by velocity gradients in a single-phase flow.

\subsection{Diverging flows}

We next turn our attention to the flow behavior of the configuration shown in Fig. 5(d) further downstream in the diverging channel. When the inlet flow is stable (i.e., $d / h<0.33$ ), typical morphologies consist of a central diffusive region made of $F$ with asymmetrical thread folding of $V$ in a side flow of $M$ [Fig. 7]. The disparity in viscosity between $F$ and $M$ produces a significant velocity difference between the fast central region and the slow side-flow. The velocity difference between each of the sheath flows prevents threads from folding at the $V-F$ interface (i.e., inward towards the channel's centerline) and the folding

(a)
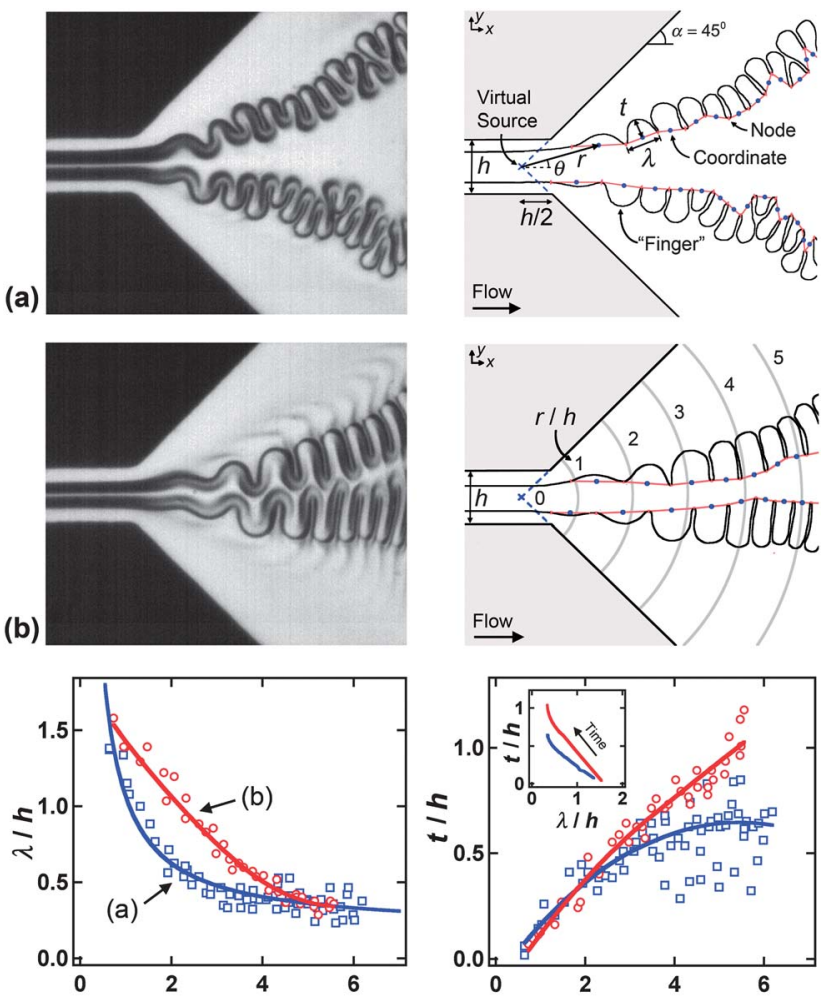

(c)

$r / h$

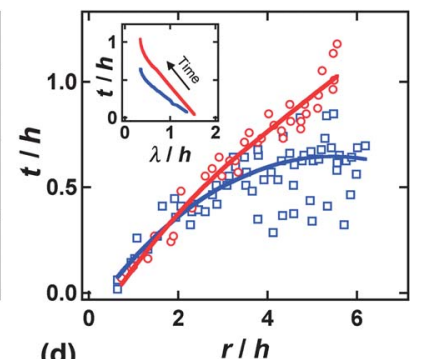

(d)

Fig. 7 Examples of intermittent flow morphologies in the diverging channel region for stable inlet flows fixed at $Q_{1}=15, Q_{2}=10$, and $Q_{3}=$ 25 in $\mu 1 \min ^{-1}$ : (a) Dendrite flow (observed: left; schematic: right), and (b) Fishbone flow (observed: left; schematic: right). (c) Evolution of the dimensionless wavelength $\lambda / h$ as a function of normalized radius $r / h$ from a virtual source at the end of the square microchannel: dendrite ( $\square)$, solid line fits: $\lambda / h=0.18+0.88(r / h)^{-1}$; fishbone $(\bigcirc)$, solid line: smoothed values. (d) Spatial evolution of the dimensionless finger length $t / h$. Inset: dimensionless length versus dimensionless wavelength (lines: smoothed values from experimental data). phenomenon remains restricted to the $V-M$ interface (i.e., outward from the channel's centerline). Although threads fold asymmetrically with a deformation that does not propagate completely through the fast central stream, threads remain hydrodynamically coupled (i.e., phase-locked) and form, along with the low-viscosity fluid, an effective structure, which regularly undulates. In addition, the highly diffusive behavior of the low-viscosity oil $F$ tends to blur the thread interface near the channel center and blended segments of threads resemble fingers.

The convected fingering patterns display a relatively complex intermittent flow behavior. In particular, although the inlet conditions are steady, we observe a feedback between the initial thread-thread separation $d$ in the square channel and the flow patterns seen in the diverging channel. For a given set of flow rates, the system displays bistability between "dendrite" flow regimes for larger $d$ [Fig. 7(a)] and "fishbone" flow regimes for smaller $d$ [Fig. 7(b)]. The dendrite flow shows finger-like protrusions that can branch along the interface between the fluids. The fishbone flow shows an alternating arrangement of unbranched finger-like protrusions that is linked to the alternating folds of the folding instability. A large-scale buckling of the entire structure made of $V$ and $F$ characterizes the transition between regimes.

We quantitatively compare patterns by measuring the apparent finger wavelength $\lambda$ and length $t$ as a function of the radius $r$ from the virtual source, which is located at $(x, y)=(-h /$ $2,0)$ from the junction between square and diverging channels. A straight line is first digitally drawn between each finger node. The center of the line defines the polar coordinates of the associated finger $(r, \theta)$, the length of the line corresponds to the finger wavelength $\lambda$, and the finger length $t$ is the distance from the finger apex to its coordinate [Fig. 7(a) - right]. As expected for a source flow in a sector, the finger wavelength $\lambda$ is overall inversely proportional to the distance $r$ [Fig. 7(c)]. The finger length $t$ is observed to increase along the flow direction [Fig. 7(d)]. For $r / h>$ 4 , the significant scatter in data during dendrite flow results in part from the lateral buckling of fingers that produce branched structures. In these graphs, the general behavior is indicated with solid trend lines that permit the average length $t$ to be related to the main wavelength $\lambda$ for both cases [see Fig. 7(d) - inset]. Experimental findings suggest a linear relationship between $t$ and $\lambda$, which is in overall agreement with the idea of the mass conservation in a finger experiencing a linear rate of deformation, as would be expected for a solenoidal velocity field, ${ }^{71}$ since diffusion and compressibility effects can be neglected as a first order approximation.

The viscous fingers that appear in the diverging channel region (i.e. for $2 \leq r / h \leq 6$ ) display a broad range of morphologies depending on initial thread size, fluid viscosities, and flow rates [Fig. 8]. Since the fingers are oriented normal to the thread's local flow direction, defined by the angle $\theta$, the decelerating flow field produces an effective lateral compression of the overall structure that can expand into multiple dendrite layers. For instance, Fig. 8(a) shows the resulting growth of one, two, and three layers of relatively large fingers. The flow pattern, here, suggests the shape of a "cactus". During the complex buckling of the metastructure formed by fluids $V$ and $F$, medium-sized fingers can deform with a large aspect ratio [Fig. 8(b)]. In this case, we label the flow morphology as "forest". Finally, thin threads separated 


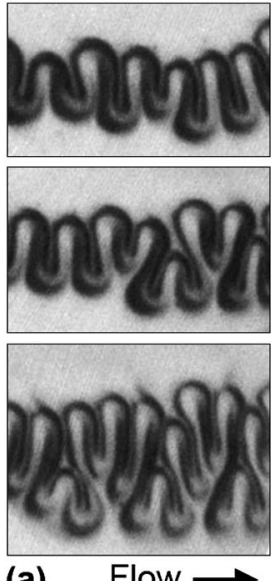

(a)

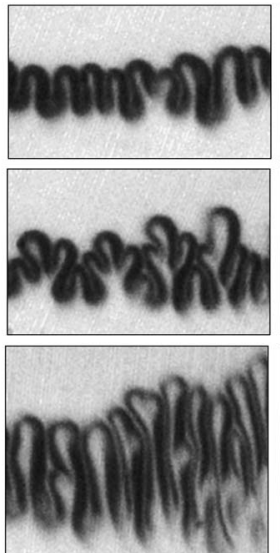

(b)

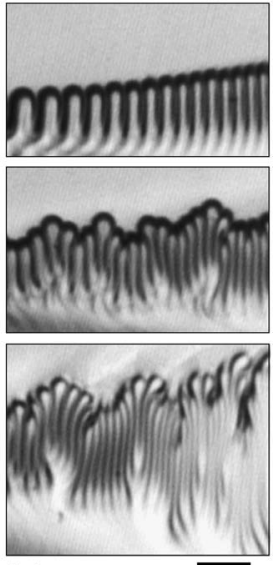

(c)
$50 \mu \mathrm{m}$

Fig. 8 Detailed examples of the typical evolution of viscous dendrites from upstream to downstream regions of a diverging channel (arranged in columns from top to bottom). Images are oriented along $\theta$, the local flow direction. Finger growth results from convective deceleration (flow rates are in $\mu 1 \mathrm{~min}^{-1}$ ): (a) "Cactus" flows exhibiting branched dendrites for thicker threads, $Q_{1}=15, Q_{2}=10, Q_{3}=25$. (b) "Forest" flows for medium-size threads, $Q_{1}=10, Q_{2}=5, Q_{3}=20$. (c) "Cilia" flows for thin threads, top image: $Q_{1}=0.5, Q_{2}=5, Q_{3}=20$; bottom two images: $Q_{1}=$ $10, Q_{2}=15, Q_{3}=55$.

by a small distance $d$ can develop into compact and very large aspect ratio arrangements than can buckle into multiple branches. Such shapes are reminiscent of "cilia" [Fig. 8(c)].

In all cases, it is important to consider that the formation of multiple layered viscous dendrites in our system results from the asymmetrical buckling of threads. The resulting morphologies suggest those observed during the Saffman-Taylor fingering instability, chemically induced growth processes, and solidification fronts. Our 2D observations do not provide a full 3D view of the compositions of the flowing fluids in the diverging channel region, so any interpretation of the visualized flow patterns necessarily involves making extrapolations that are speculative. Despite this caveat, one possible interpretation of the complex flow patterns in Fig. 7 involves a fingering instability interacting with a folding instability. Since the centrally injected flow is the least viscous liquid, the visible boundaries of flow fronts emerging from the central region in the diverging channel may finger as the least viscous liquid is driven by the extensional flows (roughly perpendicular to the deceleration along the flow direction) into the more viscous liquids outside. The relatively thin thread-like regions of highest viscosity liquid provide good refractive-index contrast as a marker for these flow fronts. However, as the fingering penetration of the least viscous liquid begins to occur, two threads of the largest viscosity liquid are also folding, thereby creating a modulation of the fingering front. This modulation is especially evident in the alternating fishbone pattern [Fig. 7(b)]; the tendency of viscous fingers to branch in diverging flows is more evident in the dendrite pattern [Fig. 7(a)]. Although this combination of instabilities is only one possible interpretation, it highlights the general idea that two or more viscous instabilities can be combined to produce interesting and complex flow patterns in microfluidic devices. A closely related phenomenon occurs during the folding of viscous layers in

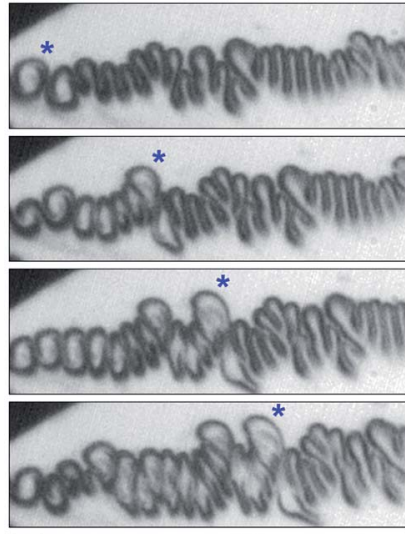

(a)

$$
\text { Flow }
$$

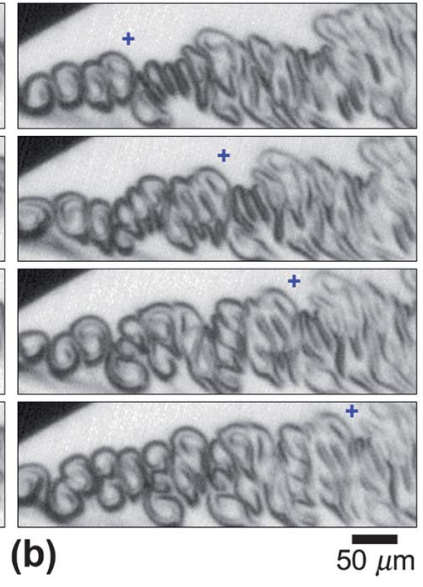

Fig. 9 Time-series of details of the swirls that form in a diverging channel and propagate downstream (time between successive frames is $6.25 \mathrm{~ms}$ ). The markers * and + identify evolution of specific swirls, flow

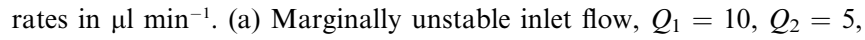
$Q_{3}=20$; dendrites are sheared, yielding some swirling features. (b) Wellformed viscous swirling flows, $Q_{1}=20, Q_{2}=10, Q_{3}=25$.

dissimilar media, which has been theoretically examined in the context of structural geology. ${ }^{78}$ The presence of walls and complex velocity fields in our case, however, prevents a direct comparison with this case.

For effective thread distances above the critical value $d / h>$ 0.33 , arrays of viscous swirls appear along the threads in the square inlet microchannel. In this case, swirls can compete with the "dendrite instability" previously discussed in the diverging channel. Fig. 9 shows two sets of images obtained from highspeed movies for the cases when (a) the flow is marginally unstable, i.e., a few swirls appears along the threads $(d / h \sim 0.33)$ and when (b) swirls are well developed along the threads $(d / h \sim$ 0.36 ). Such flows lead to very complex dynamics since viscous swirls are not necessarily all detached from one another before they enter the diverging channel. In both case, the decelerating flow produce an alternate arrangement of swirls, similar to droplets formed between immiscible liquids in diverging channels. ${ }^{23}$ Here, since the fluids are miscible, structures made of viscous fingers and swirls become compressed along the flow direction in the divergence and form complex diffusive architectures, which nevertheless remain distinguishable over short time periods using bright-field microscopic imaging.

Finally, for large thread distances, $d / h>0.6$, the inlet flow in the square channel becomes rather complex and the resulting patterns observed in the diverging channel display chaotic behavior. In this case, the proportion of low-viscosity oil $F$ is large compared to $M$ and $V$ [i.e., $\left.\alpha_{1}=Q_{1} /\left(Q_{1}+Q_{2}+Q_{3}\right)>0.5\right)$ and the central stream occupies most of the diverging channel. As a result, viscous swirls become rapidly surrounded with $F$, and a wide range of "turbid" flow patterns is observed. Fig. 10 shows a few examples of these structures composed of (a) assemblies of interacting swirls that vaguely resemble van Karman vortex streets, (b) multiple diffusive swirls joined together in complex manifolds, and (c) viscous heterogeneous flows composed of $F$, $M$, and $V$.

Overall, the formation of such highly unstable microflows using three Newtonian fluids having different viscosities suggests 


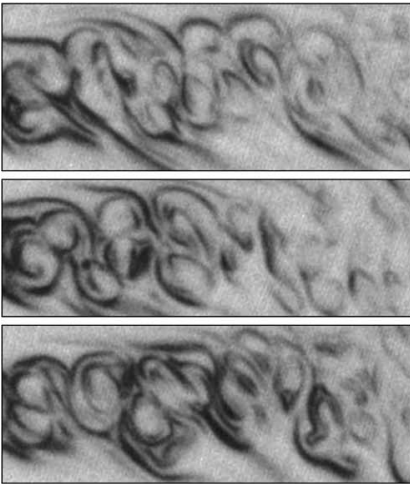

(a)

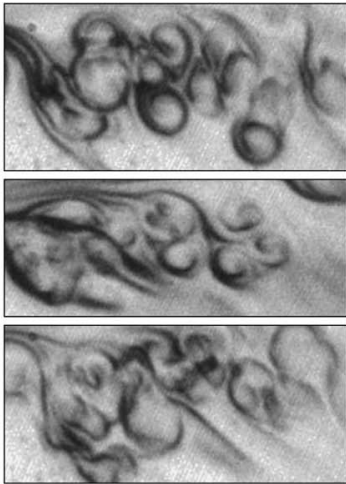

(b)

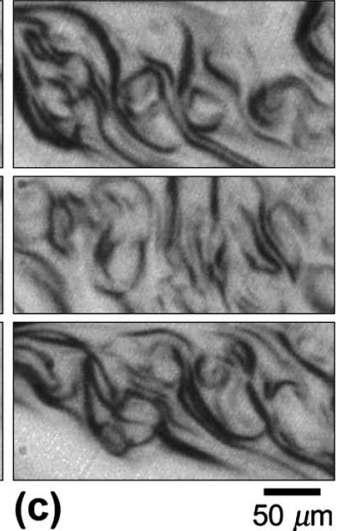

Fig. 10 Examples of chaotic miscible flow structures in the diverging channel region for large thread-thread separations $d$, flow rates in $\mu 1$ min ${ }^{-1}$. Three examples are shown at each set of flow rates. (a) $Q_{1}=50, Q_{2}=10, Q_{3}=25$. (b) $Q_{1}=100, Q_{2}=5, Q_{3}=15$. (c) $Q_{1}=200, Q_{2}=10, Q_{3}=25$.

an analogy with hydrodynamic turbulence at large Re and elastic turbulence at low Re. Indeed, these unstable flows are characterized by highly irregular spatio-temporal structures and enhanced mixing. ${ }^{79}$ As the term "viscous turbulence" however would be extremely misleading, for clarity, we prefer the term "viscous heterogeneous flows" (or "viscous chaotic flows") owing to the fact that many of the phenomena observed are based on the initial flow structures and the evolution of the local viscosity as fluid mix in the system. Here, we show that non-linear deformation processes of miscible interfaces through folding and swirling instability in microgeometries qualitatively enhance mixing of Newtonian fluids at low Re. Further work is required to quantitatively define the efficiency of mixing of differingviscosity miscible fluids in microfluidic systems.

\section{Conclusion}

In this perspective on the formation, structure, and dynamics of miscible multiphase flows of differing-viscosity fluids in microfluidics, we have emphasized how different fluid injection scenarios and geometries can be used to produce lubricated threads, viscosity-stratified flows, or combinations of both. The control of lubricated threads offer a new route for manipulating thick substances at the microscale. We have described basic thread instabilities, including folding and swirling instabilities, as well as more complex flow patterns that result from the interaction of two or more basic instabilities. Insights gained from studies of two-fluid flows have enabled us to infer morphologies of three-fluid flows in parallel and extensional geometries. Moreover, for the case study of a three-fluid flow in the diverging channel region, it is likely that the alternating undulations, resulting from the folding instability of the two highly viscous threads, play an important role in setting the initial conditions for penetration of the least viscous liquid into the more viscous liquids via a second instability - the fingering instability.

This work provides a mere glimpse into the broad and largely unexplored realm of interacting fluid instabilities, particularly viscous instabilities which can be readily produced in microfluidic devices. In the three-fluid case, we show the existence of specific injection scheme that leads to intricate flow behaviors. In particular, the transition from ordered to disordered flows is achieved by simply increasing the flow rate of the less viscous central stream. The development of secondary instabilities for three-fluid flows suggests that introducing miscible additives having different viscosities in the appropriate flow configuration may help achieve rapid mixing at the small scale.

Beyond creating new experiments to further explore a wide variety of interacting fluid instabilities, many important theoretical challenges remain. An example of a simpler scenario would be solving coupled time- and space-dependent non-linear partial differential equations for the evolving velocity and viscosity fields, which incorporate aspects of convection and diffusion subject to boundary conditions that reflect injection flow-rate conditions and wall surface properties. Much higher levels of complexity in the dynamical equations of motion for the flowing material and its local compositional changes are of course possible. For instance, in some cases, soft materials exhibiting non-Newtonian rheological properties over certain ranges of compositions could dramatically alter the dynamical pattern formation.

A complete set of rules for predicting the dominance of a given instability over another, depending upon materials, geometry, and flow conditions, has yet to be developed. Some instabilities do not occur below a well-defined "critical" onset value of a dimensionless parameter, based on the evolutionary equations, whereas other instabilities do not exhibit a critical onset. Thus, competition between different instabilities may be seen only in certain regimes of control parameters; in other regimes, the dominance of a particular instability may be very nearly complete, thereby suppressing any others. Moreover, beyond onset criteria, growth rates of two or more instabilities could be different, and these growth rates could also play an important role in controlling dominance of one type of instability over another. For instance, one instability may have an earlier onset but slower growth, thereby dominating upstream in the microchannel, whereas a second instability may have a larger growth rate, thereby enabling it to out-compete and dominate over the first instability downstream. Moreover, the first instability could provide a modulation or envelope which affects the patterns formed by the second instability downstream.

An important experimental challenge is obtaining fully threedimensional high-speed volume movies of interacting 
instabilities, even as they occur in quasi-two-dimensional "sandwich" geometries. Although interacting instabilities can be highly complex even for simple incompressible Newtonian liquids, the introduction of non-Newtonian and anisotropic liquid materials in controlled microfluidic geometries would add yet further possibilities for tuning and altering the nature of interacting instabilities.

\section{References}

1 L. Patterson, J. Fluid Mech., 1981, 113, 513.

2 P. L. Tang, E. D. Sudol, C. A. Silebi and M. S. El-Aasser, J. Appl. Polym. Sci., 1991, 43, 1059.

3 A. Rodger, Methods Enzymol., 1993, 226, 232.

4 T. G. Mason and J. Bibette, Langmuir, 1997, 13, 4600-4613.

5 A. Prigent, G. Gregoire, H. Chate, O. Dauchot and W. van Saarloos, Phys. Rev. Lett., 2002, 89, 014501.

6 S. Lerouge, M. A. Fardin, M. Argentina, G. Gregoire and O. Cardoso, Soft Matter, 2008, 4, 1808-1819.

7 C. H. Barnett and W. Cochrane, Nature, 1956, 177, 740-742.

8 B. Legait, J. Colloid Interface Sci., 1983, 96, 28-38.

9 J. Bico and D. Quere, J. Fluid Mech., 2002, 467, 101-127.

10 M. J. Madou, Fundamentals of Macrofabrication and Nanotechnology, CRC Press, Boca Raton, FL, 2012, vol. II.

11 T. H. Wang, Y. H. Peng, C. Y. Zhang, P. K. Wong and C. M. Ho, J. Am. Chem. Soc., 2005, 127, 5354-5359.

12 K. K. Liu, R. G. Wu, Y. J. Chuang, H. S. Khoo, S. H. Huang and F. G. Tseng, Sensors, 2010, 10, 6623-6661.

13 T. M. Squires and S. R. Quake, Rev. Mod. Phys., 2005, 77, 977-1026.

14 N.-T. Nguyen and S. T. Wereley, Fundamentals and Applications of Microfluidics, Artech House Inc., Norwood, 2006.

15 W. Drenckhan, S. J. Cox, G. Delaney, H. Holste, D. Weaire and N. Kern, Colloids Surf., A, 2005, 263, 52-64.

16 E. Lorenceau, Y. Y. C. Sang, H. Reinhard and S. Cohen-Addad, Phys. Fluids, 2006, 18, 097103.

17 J. P. Raven and P. Marmottant, Phys. Rev. Lett., 2009, 102, 084501.

18 D. R. Link, S. L. Anna, D. A. Weitz and H. A. Stone, Phys. Rev. Lett., 2004, 92, 054503.

19 L. Ménétrier-Deremble and P. Tabeling, Phys. Rev. E: Stat., Nonlinear, Soft Matter Phys., 2006, 74, 035303(R).

20 T. Cubaud, Phys. Rev. E: Stat., Nonlinear, Soft Matter Phys., 2009, 80, 026307.

21 Y. C. Tan, Y. L. Ho and A. P. Lee, Microfluid. Nanofluid., 2007, 3, 495.

22 N. Bremond, A. R. Thiam and J. Bibette, Phys. Rev. Lett., 2008, 100, 024501.

23 B. M. Jose and T. Cubaud, Microfluid. Nanofluid., 2012, 12, 687.

24 R. K. Shah, H. C. Shum, A. C. Rowat, D. Lee, J. J. Agresti, A. S. Utada, L. Y. Chu, J. W. Kim, A. Fernandez-Nieves, C. J. Martinez and D. A. Weitz, Mater. Today, 2008, 11, 18.

25 N. Pannacci, H. Bruss, D. Bartolo, I. Etchart, T. Lockhart, Y. Hennequin, H. Willaime and P. Tabeling, Phys. Rev. Lett., 2008, 101, 164502.

26 S. A. Khan and S. Duraiswamy, Lab Chip, 2009, 9, 1840.

27 P. G. Saffman and G. Taylor, Proc. R. Soc. London, Ser. A, 1958, 245, 312.

28 J. S. Langer, Science, 1989, 243, 1150-1156.

29 G. M. Homsy, Annu. Rev. Fluid Mech., 1987, 19, 271-311.

30 J. Nittmann, G. Daccord and H. E. Stanley, Nature, 1985, 314, 141144.

31 E. Pitts, J. Fluid Mech., 1980, 97, 53.

32 P. Garstecki, H. A. Stone and G. W. Whitesides, Phys. Rev. Lett., 2005, 94, 164501.

33 P. Guillot, A. Colin, A. S. Utada and A. Ajdari, Phys. Rev. Lett., 2007, 99, 104502.

34 T. Cubaud and T. G. Mason, Phys. Fluids, 2008, 20, 053302.

35 A. Groisman, M. Enzelberger and S. R. Quake, Science, 2003, 300, 955-958.

36 T. Burghelea, E. Segre, I. Bar-Joseph, A. Groisman and V. Steinberg, Phys. Rev. E: Stat., Nonlinear, Soft Matter Phys., 2004, 69, 066305.
37 R. J. Poole, M. A. Alves and P. J. Oliveira, Phys. Rev. Lett., 2007, 99, 164503.

38 O. Bonhomme, A. Morozov, J. Leng and A. Colin, Phys. Rev. E: Stat., Nonlinear, Soft Matter Phys., 2011, 83, 065301(R).

39 J. Wang and D. F. James, J. Rheol., 2011, 55, 1103.

40 C. P. Pipe and G. H. McKinley, Mech. Res. Commun., 2009, 36, 110. 41 D. Di Carlo, Lab Chip, 2009, 9, 3038.

42 X. Mao, J. R. Waldeisen and T. J. Huang, Lab Chip, 2007, 7, 1260.

43 J. M. Martel and M. Toner, Phys. Fluids, 2012, 24, 032001.

44 M. A. Holden, S. Kumar, E. T. Castellana, A. Beskok and P. S. Cremer, Sens. Actuators, B, 2003, 92, 199-207.

45 J. Goulpeau, B. Lonetti, D. Trouchet, A. Ajdari and P. Tabeling, Lab Chip, 2007, 7, 1154-1161.

46 W. Georgescu, J. Jourquin, L. Estrada, A. R. A. Anderson, V. Quanrata and J. P. Wikswo, Lab Chip, 2008, 8, 238-244.

47 J. B. Knight, A. Vishwanath, J. P. Brody and R. H. Austin, Phys. Rev. Lett., 1998, 80, 3863-3866.

48 R. F. Ismagilov, A. D. Stroock, P. J. A. Kenis, G. Whitesides and H. A. Stone, Appl. Phys. Lett., 2000, 76, 2376.

49 J. Jimenez, J. Fluid Mech., 2005, 535, 245-254.

50 Z. G. Wu and N. T. Nguyen, Sens. Actuators, B, 2005, 107, 965974.

51 G. B. Lee, C. C. Chang, S. B. Huang and R. J. Yang, J. Micromech. Microeng., 2006, 16, 1024-1032.

52 J. Dambrine, B. Géraud and J.-B. Salmon, New J. Phys., 2009, 11, 075015.

53 C. Y. Lee, C. L. Chang, Y. N. Wang and L. M. Fu, Int. J. Mol. Sci., 2011, 12, 3263-3287.

54 J. M. Ottino, Annu. Rev. Fluid Mech., 1990, 22, 207.

55 S. Wang, X. Huang and C. Yang, Lab Chip, 2011, 11, 2081.

56 H. M. Xia, Z. P. Wang, Y. X. koh and K. T. May, Lab Chip, 2010, 10, 1712.

57 T. Cubaud and T. G. Mason, Phys. Rev. Lett., 2006, 96, 114501.

58 D. D. Joseph, K. Nguyen and G. S. Beavers, J. Fluid Mech., 1984, 141, 319-345.

59 D. D. Joseph and Y. Y. Renardy, Fundamentals of Two-Fluid Dynamics. Part II: Lubricated Transport, Drops and Miscible Liquids, Springer-Verlag, New York, 1993.

60 B. Khomami and K. C. Su, J. Non-Newtonian Fluid Mech., 2000, 91, $59-84$.

61 M. Skorobogatiy and L. Mahadevan, Europhys. Lett., 2000, 52, 532538.

62 S. Chiu-Webster and J. R. Lister, J. Fluid Mech., 2006, 569, 89-111.

63 N. M. Ribe, M. Habibi and D. Bonn, Annu. Rev. Fluid Mech., 2012, 44, 249.

64 T. Cubaud and T. G. Mason, Phys. Rev. Lett., 2007, 98, 264501.

65 F. Ould-Kaddour and D. Levesque, Phys. Rev. E: Stat. Phys., Plasmas, Fluids, Relat. Interdiscip. Top., 2000, 63, 011205.

66 T. Cubaud and T. G. Mason, Phys. Rev. E: Stat., Nonlinear, Soft Matter Phys., 2008, 78, 056308.

67 D. J. Tritton, Physical Fluid Dynamics, Oxford University Press Inc., New York, 1988.

68 E. Guyon, J.-P. Hulin, L. Petit and C. D. Mitescu, Physical Hydrodynamics, Oxford University Press, New York, 2001.

69 T. Cubaud and T. G. Mason, New J. Phys., 2009, 11, 075029.

70 T. Cubaud, B. M. Jose, S. Darvishi and R. Sun, Int. J. Multiphase Flow, 2012, 39, 29.

71 G. K. Batchelor, An Introduction to Fluid Mechanics, Cambridge University Press, New York, 1967.

72 P. Guillot, P. Panizza, J. B. Salmon, M. Joanicot, A. Colin, C. H. Bruneau and T. Colin, Langmuir, 2006, 22, 6438-6445.

73 J. T. Cabral and S. D. Hudson, Lab Chip, 2006, 6, 427-436.

74 T. Cubaud, B. M. Jose and S. Darvishi, Phys. Fluids, 2011, 23, 042002.

75 S. Darvishi and T. Cubaud, J. Fluids Eng., 2011, 133, 031203.

76 L. D. Landau and E. M. Lifshitz, Theory of Elasticity, Pergamon Press, Oxford, 1986.

77 T. Cubaud and T. G. Mason, Phys. Fluids, 2006, 18, 091108.

78 A. M. Johnson and R. C. Fletcher, Folding of Viscous Layers, Columbia University Press, New York, 1994.

79 P. Huerre and M. Rossi, in Hydrodynamics and Nonlinear Instabilities, ed. G. Godrèche and P. Manneville, Cambridge University Press, Cambridge, UK, 2005. 